\title{
An optimized fully-passive noise-shaping SAR ADC with integration capacitor reuse technique
}

\author{
Yi Yang ${ }^{1}$, Di Li ${ }^{1 \text {, a) }}$, Chanrong Jiang ${ }^{1}$, Yuqian Liu ${ }^{1}$, and Yintang Yang ${ }^{1}$
}

Abstract This paper presents an optimized Fully-Passive Noise-Shaping Successive Approximation Register (FPNS-SAR) Analog-to-Digital Converter (ADC) with passive gain and integration capacitor reuse techniques. Instead of using multi-input-pair comparator with active gain in traditional Cascaded Integrator Feed-Forward (CIFF) structure, 1-input-pair comparator with passive gain is adopted in this paper to reduce power consumption and kick-back noise. The proposed FPNS-SAR ADC was implemented with standard 65-nm CMOS process. With the oversampling ratio (OSR) of 8 , the simulation results realize $79.3 \mathrm{~dB}$ peak SNDR and $94 \mathrm{~dB}$ peak SFDR at $6.25 \mathrm{MHz}$ input signal bandwidth and $100 \mathrm{MHz}$ sampling frequency. The proposed ADC consumes $1.245 \mathrm{~mW}$ at $1.2 \mathrm{~V}$ supply voltage. The calculated FOMw and FOMs are $13.2 \mathrm{fJ} /$ conv.-step and $176.3 \mathrm{~dB}$, respectively.

Keywords: Analog-to-Digital Converter (ADC), Fully-Passive NoiseShaping (FPNS), Cascaded Integrator Feed-Forward (CIFF), Successive Approximation Register (SAR)

Classification: Integrated circuits (memory, logic, analog, RF, sensor)

\section{Introduction}

The Successive Approximation Register (SAR) Analog-toDigital Converter (ADC) is widely used in moderate resolution and conversion rate due to its structure simplicity, low power consumption and friendly for process scaling. Due to the limitation of the quantization noise and thermal noise, the resolution of SAR ADC can only achieve about 10-bit $[1,2]$. Refs. [3, 4, 5, 6] had achieved a resolution greater than 12-bit with extra calibration circuit, but resulting more power consumption and lower conversion rate. In addition, the oversampling and noise shaping techniques implemented in sigma-delta ADC [7] are the effective ways to improve conversion accuracy. Nevertheless, the traditional Operational Transconductance Amplifier (OTA) based sigma-delta ADC $[8,9,10,11]$ is not a good choice in low power consumption application. To address these challenges, Ref. [12] and [13] had proposed VCO-based quantizer Sigma-Delta ADC to decrease the power consumption. However, the linearity of the VCO is sensitive to the vibration of Process Voltage-Temperature (PVT).

Recent researches had brought forward an attractive solution, the noise-shaping SAR (NS-SAR) ADC, which combines the oversampling and noise-shaping techniques with

${ }^{1}$ Wide Bandgap Semiconductor Technology Disciplines State Key Laboratory, Xidian University, Xi' an 710071, P.R. China

a) lidi2004@126.com

DOI: 10.1587/elex.17.20200193

Received June 15, 2020

Accepted July 9, 2020

Publicized July 27, 2020

Copyedited August 25, 2020
SAR ADC $[14,15,16,17,18,19,20,21,22,23]$. The active noise-shaping technique proposed in Refs. [14, 15, 16] is implemented by OTA to integrate the residue voltage, which sacrifices power efficiency and scaling compatibility to achieve a better noise transform function (NTF). Without OTA based integrator, Refs. [17, 18, 19] adopt Dynamic Amplifier (D-Amp) to cut down power consumption while maintaining noise suppression in-band simultaneously. However, they are very sensitive to PVT vibration, resulting in poor robust. Refs. [20, 21, 22, 23] has designed several fullypassive switch capacitor integrators, mainly including Cascaded Integrator Feed-Forward (CIFF) and Error-Feedback (EF) structures. As shown in Fig. 1, an active gain multiple input comparator is required in traditional CIFF NS-SAR to compensate for residual voltage loss, but it will generate additional power consumption and input-referred noise.

To address those issues, the passive gain and integration capacitor reuse techniques are introduced in the proposed fully-passive noise-shaping SAR ADC. Compared with traditional CIFF structure, the proposed design reduces the power consumption and kick back noise of the comparator, while retaining the strong noise-shaping effect simultaneously. The optimized FPNS SAR realizes 1st-order noise shaping effect and its NTF can achieve $19 \mathrm{~dB}$ in-band attenuation. Designed in 65-nm CMOS process, it obtains 79.3 dB SNDR with 10-bit Capacitive Digital-to-Analog Converter (CDAC) array. Additionally, the body effect cancellation technique is applied in bootstrap sampling switch to enhance linearity.

The rest of this paper is organized as follows. The architecture of the proposed optimized fully-passive noise-shaping SAR ADC and its conversion process are described in Section 2. The circuit implementation details are shown in Section 3. The simulation results are presented in Section 4. Finally, a conclusion is drawn in Section 5.

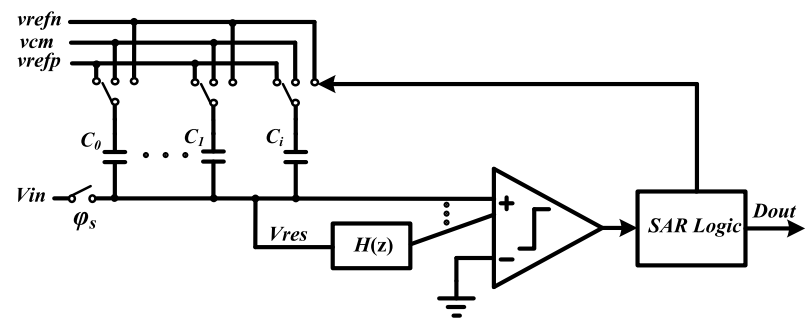

Fig. 1 The block diagram of the traditional CIFF NS-SAR. 


\section{Proposed FPNS-SAR}

The architecture of the proposed fully-passive noise-shaping SAR ADC and its time diagram are shown in Fig. 2, which is mainly composed of the CDAC array, the passive switchcapacitor integrator, the comparator and the SAR logic. Although the actual CDAC array is fully differential, for simplicity, a single-ended version is drawn here.

In the sampling phase of $\varphi_{s}$, the top plates of the CDAC array are connected to the input signal, while the bottom plates are reset to $V_{\mathrm{cm}}$. In the course of conversion, the SAR ADC carries out successive comparison which performs $V_{\mathrm{cm}}$-based switching capacitor procedure to guarantee the common voltage of the differential input $V_{\text {in }}$ and $V_{\text {ip }}$ constant [24].

After the conversion phase of $\varphi_{\mathrm{c}}$, the residual voltage $V_{\text {res }}$ is generated by returning the LSB's decision to the CDAC array, which is boosted twice by passive gain technique. Fig. 3 demonstrates the process of the CDAC to achieve passive gain, where $C_{\mathrm{MSB}}$ is the most significant capacitance and the $C_{\mathrm{LSB}}$ represents all the remaining capacitance.

Fig. 4(a) shows the conversion process of the 1 st-order noise-shaping performed by the passive switch-capacitor filter in the integration phase $\varphi 1$ and its single-ended equivalent circuit is shown in Fig. 4(b). Due to the charge redistribution between the CDAC capacitor and the integration capacitor, the residual voltage $V_{\text {res }}^{\prime}$ changes as described in Eq. (1).

$$
4 C V_{\text {int }}(n-1)+C V_{\text {res }}^{\prime}(n)=5 C V_{\text {int }}(n)
$$

Where $C$ is the total capacitance of the CDAC. The expression in z-domain is shown in Eq. (2).

$$
V_{\text {int }}(z)=\frac{0.2}{1-0.8 z^{-1}} V_{\text {res }}^{\prime}(z)
$$

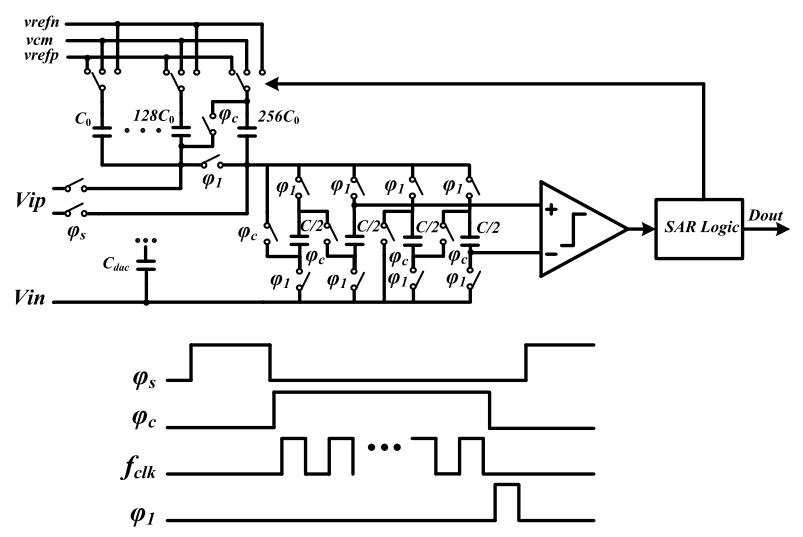

Fig. 2 Architecture of the proposed FPNS-SAR and its time diagram.

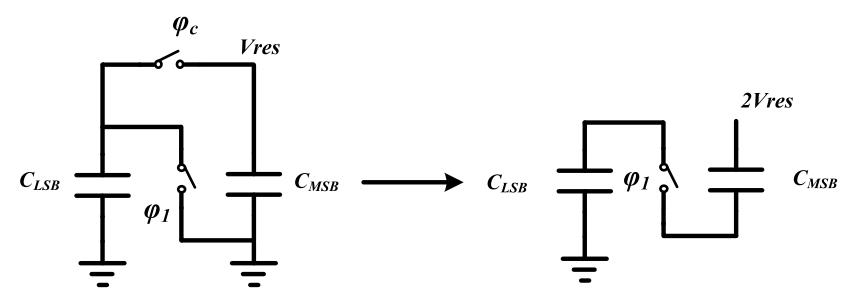

Fig. 3 Passive gain of the CDAC.
The 1st-order noise-shaping is completed. In the next conversion phase of $\varphi_{\mathrm{c}}$, the integrated residual voltage $V_{\text {int }}(z)$ will be boosted to 4 times through capacitor passive addition method. Meanwhile, the voltage of the comparator input equal to $V_{\text {res }}^{\prime}(n)+4 V_{\text {int }}(n)$. Compared to 2-input-pair, the power consumption of the comparator has been cut down by using passive addition method. The equivalent circuit of the integrated capacitor reuse technique is illustrated in Fig. 5.

The signal flow diagram of the proposed FPNS-SAR is shown in Fig. 6. Based on Eq. (2), the passive integration function can be expressed as

$$
H(z)=\frac{0.2}{1-0.8 z^{-1}}
$$

From the signal flow diagram, the expression of the digital output has been calculated.

$$
D_{\text {out }}(z)=V_{\text {in }}(z)+4 V_{\text {int }}(z)+Q(z)
$$

By analyzing the signal flow diagram and combining with Eq. (4), the transfer function of the proposed FPNS-SAR can be expressed as

$$
D_{\text {out }}(z)=V_{\text {in }}(z)+\frac{1-0.8 z^{-1}}{1+0.8 z^{-1}} Q(z)
$$

From Eq. (5), the noise transform function can be calculated as

$$
\operatorname{NTF}(z)=\frac{1-0.8 z^{-1}}{1+0.8 z^{-1}}
$$

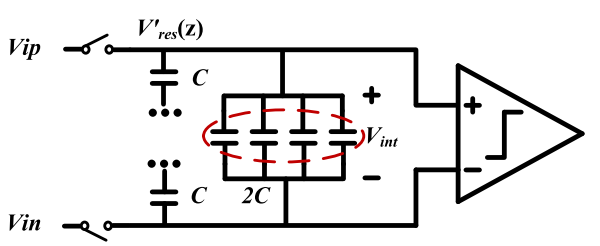

(a)

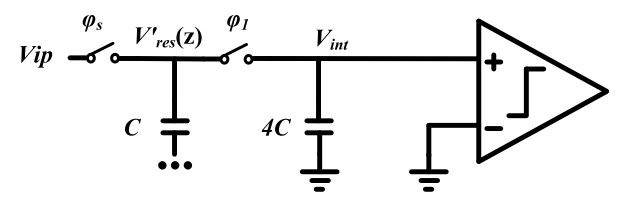

(b)

Fig. 4 The $1^{\text {st }}$-integration process.

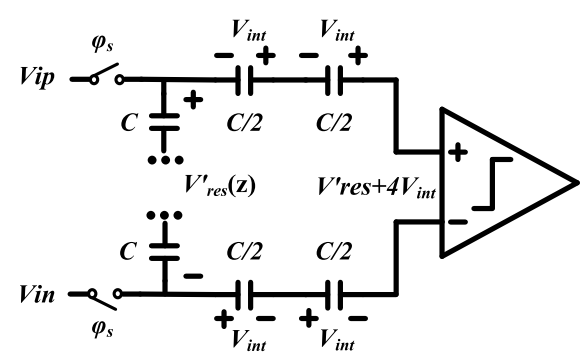

Fig. 5 The passive addition and integration capacitor reuse.

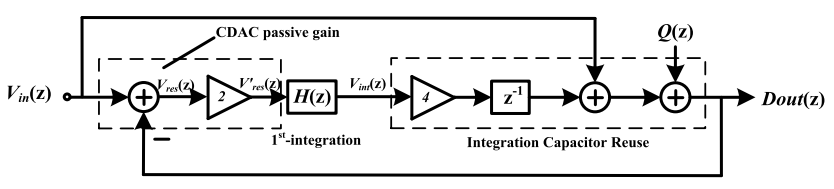

Fig. 6 General signal flow diagram of the proposed FPNS SAR ADC. 


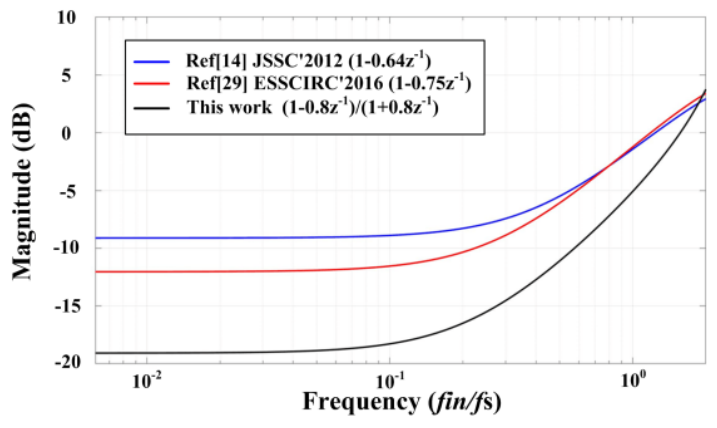

Fig. 7 Bode-diagrams comparison in this paper and prior works.

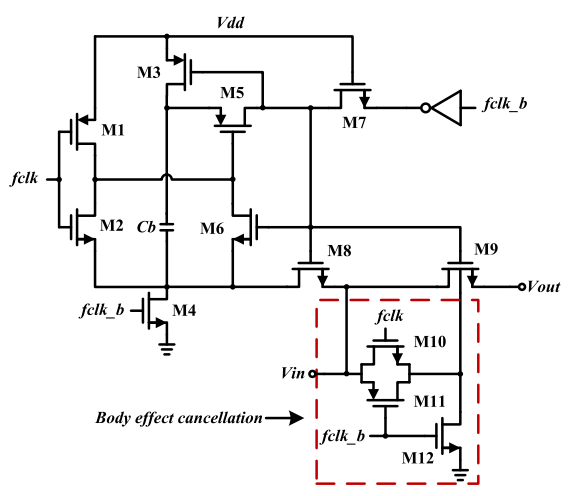

Fig. 8 Implementation of the bootstrap switch.

which contains one zero located in $z=0.8$ and one pole located in $z=-0.8$. The bode-diagrams comparison in this paper and prior works, shown in Fig. 7, proves that the NTF of the proposed FPNS-SAR can achieve $19 \mathrm{~dB}$ noise attenuation in signal band.

\section{Circuit implementation}

\subsection{Bootstrap switch}

The performance of the sample and hold circuit is critical for high-speed and high-precision ADCs. General NMOS switch and CMOS transmission gate are not good choices for those application with low nonlinear distortion. To reduce harmonic distortion, the bootstrap switches are adopted in ADCs [25, 26]. However, the linearity of the bootstrap switch is mainly deteriorated by the charge injection effect and body effect. In a fully differential SAR ADC, the charge injection can be eliminated by differential sampling. In order to solve the body effect, the structure of the bootstrap switch with bode effect cancellation technique is shown in Fig. 8.

$$
R_{\mathrm{on}}=\frac{1}{\mu_{n} C_{o x}(W / L)\left(V_{G S}-V_{\mathrm{th}}\right)}
$$

The calculated equivalent on-resistance of the switching transistor, $R_{\text {on }}$, is shown in Eq. (7). Due to the variable $V_{\text {th }}$ caused by body effect, the nonlinearity of the sampling switches gets worse. In this work, transistors M10, M11 and M12 are added to the bootstrap circuit to keep $V_{\text {th }}$ constant, thereby eliminating the nonlinearity.

To verify the effectiveness of this technique, Fig. 9(a) and Fig. 9(b) show the sampling signal spectrum of before body effect cancellation and after body effect cancellation, respectively. From the FFT results, the dynamic performance

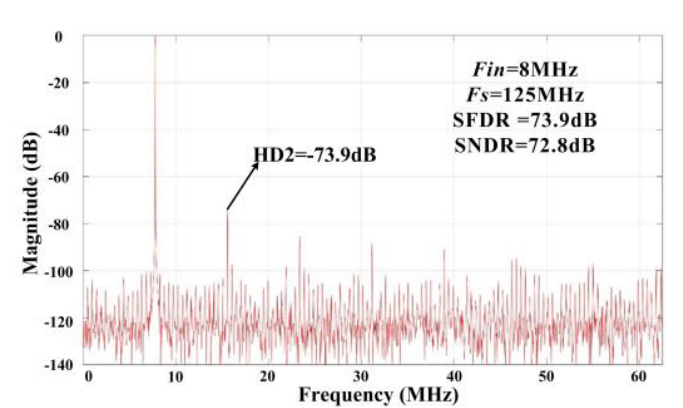

(a) Before body effect cancellation

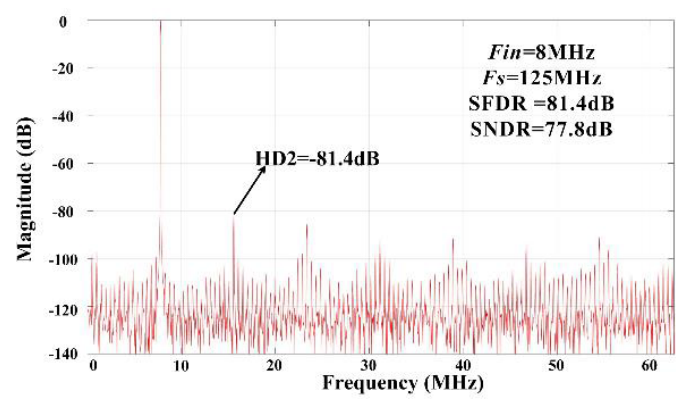

(b) after body effect cancellation

Fig. 9 Body effect cancellation technique in bootstrap switch.
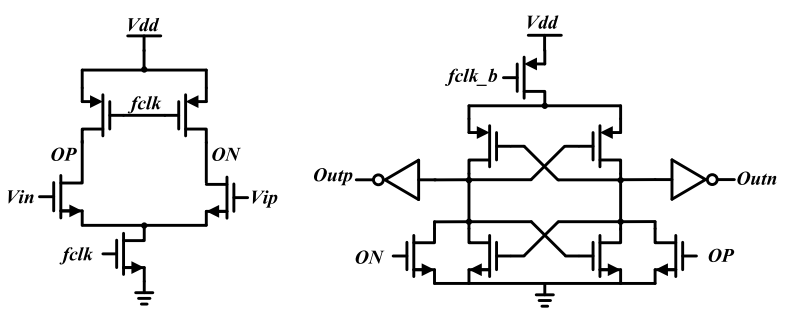

Fig. 10 The double-tail comparator.

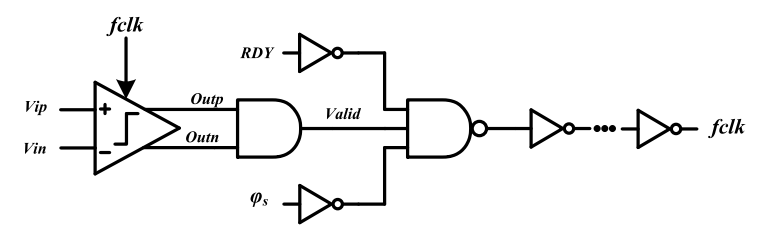

Fig. 11 Generation circuit of $f_{\text {clk }}$.

can be significantly improved with body effect cancellation technique under the condition of $8 \mathrm{MHz}$ input sine wave and $125 \mathrm{MHz}$ sampling rate. After body effect cancellation, the SNDR and SFDR increase by $5 \mathrm{~dB}$ and $7.5 \mathrm{~dB}$, respectively.

\subsection{Clock generation circuit}

Asynchronous sequential logic is used in the SAR ADC [1, $24]$ to improve the conversion rate, which reduces the requirements of the external clock and thereby the overall power consumption of the circuit.

The clock generation circuit works like an oscillator and is mainly composed of a comparator and 3-input NAND gate. As depicted in Fig. 10, the double-tail dynamic comparator controlled by $f_{\text {clk }}$ is adopted in this work to reduce the power consumption and kick-back noise of the comparator [27].

Fig. 11 shows the overall circuit implementation of the internal clock. Where, the RDY signal is the conversion completion signal, while Outp and Outn are the output sig- 


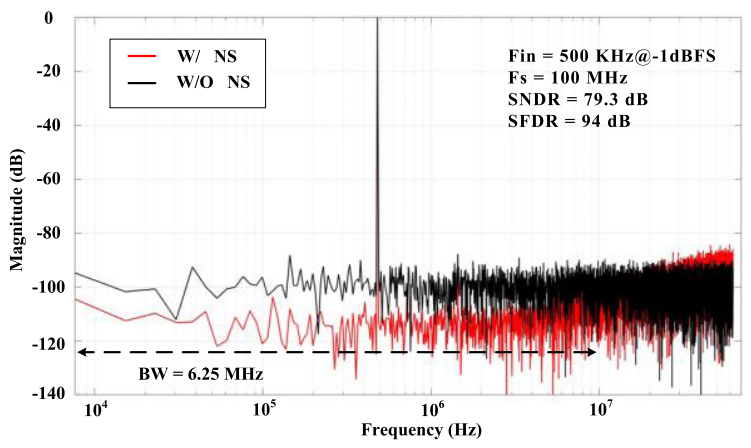

Fig. 12 Simulated FFT spectrum at $100 \mathrm{MHz}$.

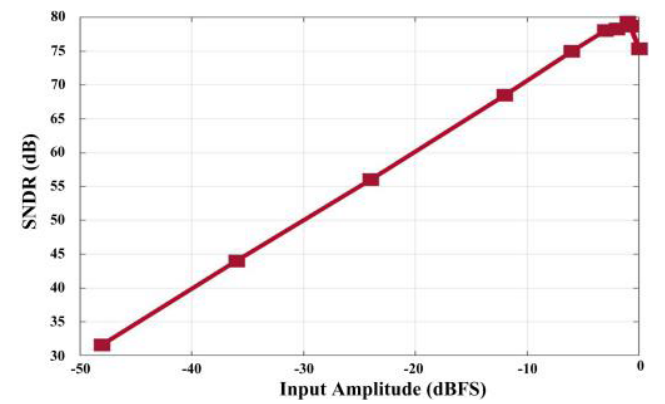

(a)

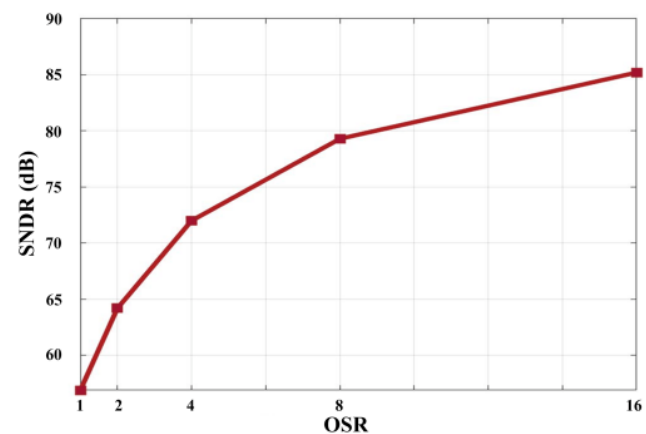

(b)

Fig. 13 Simulated SNDR versus (a) input amplitude and (b) OSR.

nals of the comparator.

\section{Results and discussion}

The proposed optimized FPNS SAR was designed in TSMC 1P8M 65-nm CMOS technology. The minimum MIM capacitance defined by this process is $9.4 \mathrm{fF}$ with an area 2 um $* 2$ um. The unit capacitance used in this paper is $4.7 \mathrm{fF}$, which is realized by two minimum process-defined MIM capacitors connected in series. With $1.2 \mathrm{~V}$ supply voltage and $100 \mathrm{MHz}$ sampling frequency, the total power consumption is $1.245 \mathrm{~mW}$.

Fig. 12 demonstrates the 16384-point Fast Fourier Transform spectrum of this work with a $-1 \mathrm{dBFS}$ sine wave at $500 \mathrm{KHz}$. The bandwidth of the input signal is $6.25 \mathrm{MHz}$ with an OSR of 8. From the measured spectrum, the ADC achieves a peak SNDR of $79.3 \mathrm{~dB}$ and a peak SFDR of $94 \mathrm{~dB}$ with $1^{\text {st }}$-order noise shaping. Furthermore, an ENOB of 12.88 bits was achieved with a 10-bit CDAC array.

Fig. 13 illustrates the simulated SNDR versus the input amplitude and the OSR. The performance comparison with
Table I Performance summary and comparison.

\begin{tabular}{|c|c|c|c|c|c|}
\hline \multirow{2}{*}{ Architecture } & $\begin{array}{l}\text { Ref[14] }{ }^{*} \\
\text { JSSC'12 }\end{array}$ & $\begin{array}{l}\text { Ref [19]* } \\
\text { IEICE'18 }\end{array}$ & $\begin{array}{l}\text { Ref[21]* } \\
\text { VLSI'17 }\end{array}$ & $\begin{array}{l}\text { Ref[30]** } \\
\text { VLSI'18 }\end{array}$ & $\begin{array}{c}\text { This } \\
\text { work** }\end{array}$ \\
\hline & $\begin{array}{c}\text { Active } \\
\text { NS SAR } \\
\end{array}$ & $\begin{array}{l}\text { D-Amp } \\
\text { NS SAR } \\
\end{array}$ & $\begin{array}{l}\text { FPNS } \\
\text { SAR }\end{array}$ & $\begin{array}{l}\text { FPNS } \\
\text { SAR } \\
\end{array}$ & $\begin{array}{l}\text { FPNS } \\
\text { SAR }\end{array}$ \\
\hline $\begin{array}{c}\text { Technology } \\
(\mathrm{nm})\end{array}$ & 65 & 65 & 40 & 65 & 65 \\
\hline Supply (V) & 1.2 & 1 & 1 & 1 & 1.2 \\
\hline $\begin{array}{l}\text { Sampling rate } \\
(\mathrm{MS} / \mathrm{s})\end{array}$ & 90 & 25 & 8.4 & 100 & 100 \\
\hline OSR & 4 & 20 & 16 & 25 & 8 \\
\hline $\begin{array}{l}\text { Bandwidth } \\
\text { (MHz) }\end{array}$ & 11 & 0.625 & 0.26 & 2 & 6.25 \\
\hline SNDR $(\mathrm{dB})$ & 62.14 & 80.4 & 80 & 82 & 79.3 \\
\hline Power $(\mathrm{uW})$ & 806 & 630.2 & 140 & 561 & 1245 \\
\hline FoMs $(\mathrm{dB})$ & 163.5 & 170.4 & 173 & 176 & 176.3 \\
\hline $\begin{array}{c}\text { FoMw } \\
\text { (fJ/conv.step) }\end{array}$ & 35.8 & 59 & 33 & 16 & 13.2 \\
\hline \multicolumn{6}{|c|}{ FOMs = SNDR $(\mathrm{dB})+10 \lg (\mathrm{BW} /$ Power $)$} \\
\hline \multicolumn{6}{|c|}{$\mathrm{FOMw}=\mathrm{Power} / 2^{\mathrm{ENOB}} * 2 * \mathrm{BW}$} \\
\hline
\end{tabular}

the prior works is presented in Table I [30].

\section{Conclusion}

To further reduce the overall power consumption of the ADC while maintaining good noise shaping effect, an optimized FPNS SAR ADC with passive gain and integration capacitor reuse techniques was proposed in this paper. It achieves a peak SNDR of $79.3 \mathrm{~dB}$ and a peak SFDR of $94 \mathrm{~dB}$ at $100 \mathrm{MHz}$ sampling frequency. Compared with prior works, a better noise-shaping effect has been realized in CIFF structure which requested only 1-input-pair comparator. Meanwhile, the body effect cancellation bootstrap switch is also adopted in this paper to achieve better linearity.

\section{Acknowledgments}

This research is supported by National Natural Science Foundation of China (Grant No. 61504102 and 11604251), the National Key Project of Intergovernmental Cooperation in International Scientific and Technological Innovation (2016YFE0107900).

\section{References}

[1] C.-C. Liu, et al.: "A 10-bit 50-MS/s SAR ADC with a monotonic capacitor switching procedure," IEEE J. Solid-State Circuits 45 (2010) 731 (DOI: 10.1109/JSSC.2010.2042254).

[2] P. Harpe, et al.: "A 10b/12b $40 \mathrm{kS} / \mathrm{s}$ SAR ADC with data-driven noise reduction achieving up to $10.1 \mathrm{~b}$ ENOB at $2.2 \mathrm{fJ} /$ conversion-step," IEEE J. Solid-State Circuits 48 (2013) 3011 (DOI: 10.1109/JSSC. 2013.2278471).

[3] W.-B. Liu, et al:: "A 12-bit, 45-MS/s, 3-mW redundant successiveapproximation-register analog-to-digital converter with digital calibration,” IEEE J. Solid-State Circuits 46 (2011) 2661 (DOI: 10.1109/ JSSC.2011.2163556).

[4] S.-B. Liu, et al.: "A 12-bit $10 \mathrm{MS} / \mathrm{s}$ SAR ADC with high linearity and energy-efficient switching," IEEE Trans. Circuits Syst. I, Reg. Papers 63 (2016) 1616 (DOI: 10.1109/TCSI.2016.2581177).

[5] T. Numata, et al.: "A 16-bit 8-MS/s SAR ADC with a foreground calibration and hybrid-charge-supply power structure," IEICE Electron. Express 10 (2013) 20200097 (DOI: 10.1587/elex.17.20200097).

[6] A. Bannon, et al.: "An 18 b 5 MS/s SAR ADC with 100.2 dB dynamic range," IEEE Symposium on VLSI Circuits Dig. Tech. Papers (2014) (DOI: 10.1109/VLSIC.2014.6858371).

[7] T. Samad, et al.: Understanding Delta-Sigma Data Converters (Wiley- 
IEEE Press, 2017) 2nd ed.

[8] A. Edward, et al.: "43-mW MASH 2-2 CT $\Sigma \Delta$ modulator attaining $74.4 \mathrm{~dB} / 75.8 \mathrm{~dB} / 76.8 \mathrm{~dB}$ SNDR/SNR/DR and $50 \mathrm{MHz}$ of BW in 40-nm CMOS," IEEE J. Solid-State Circuits 52 (2017) 448 (DOI: 10.1109/JSSC.2016.2616361).

[9] L. Qi, et al.: "A 76.6-dB-SNDR 50-MHz-BW 29.2-mW multi-bit CT sturdy MASH with DAC non-linearity tolerance," IEEE J. Solid-State Circuits 55 (2020) 344 (DOI: 10.1109/JSSC.2019.2942359).

[10] Y.-S. Kwak, et al.: "A 72.9-dB SNDR 20-MHz BW 2-2 discretetime sturdy MASH delta-sigma modulator using source-followerbased integrators," IEEE J. Solid-State Circuits 53 (2018) 2772 (DOI: 10.1109/JSSC.2018.2859401).

[11] S.-H. Wu, et al.: "A 81-dB dynamic range 16-MHz bandwidth $\Delta \Sigma$ modulator using background calibration," IEEE J. Solid-State Circuits 48 (2013) 2170 (DOI: 10.1109/JSSC.2013.2264137).

[12] M.Z. Straayer, et al:: "A 12-bit, 10-MHz bandwidth, continuoustime ADC with a 5-Bit, 950-MS/s VCO-based quantizer," IEEE J. Solid-State Circuits 43 (2008) 814 (DOI: 10.1109/JSSC.2008. 917500).

[13] S.-L. Li, et al.: "A 174.3-dB FoM VCO-based CT $\Delta \Sigma$ modulator with a fully-digital phase extended quantizer and tri-level resistor DAC in 130-nm CMOS," IEEE J. Solid-State Circuits 52 (2017) 1940 (DOI: 10.1109/JSSC.2017.2693244).

[14] J.A. Fredenburg, et al.: "A 90-MS/s 11-MHz-bandwidth 62-dB SNDR noise-shaping SAR ADC," IEEE J. Solid-State Circuits 47 (2012) 2898 (DOI: 10.1109/JSSC.2012.2217874).

[15] K. Obata, et al.: "A $97.99 \mathrm{~dB}$ SNDR, $2 \mathrm{kHz}$ BW, $37.1 \mu \mathrm{W}$ noiseshaping SAR ADC with dynamic element matching and modulation dither effect," IEEE VLSI Circuits (2016) (DOI: 10.1109/VLSIC. 2016.7573463).

[16] Y.-S. Shu, et al.: "An oversampling SAR ADC with DAC mismatch error shaping achieving $105 \mathrm{~dB}$ SFDR and $101 \mathrm{~dB}$ SNDR over $1 \mathrm{kHz}$ BW in 55 nm CMOS," IEEE J. Solid-State Circuits 51 (2016) 2928 (DOI: 10.1109/JSSC.2016.2592623).

[17] C.-C. Liu, et al.: "A $0.46 \mathrm{~mW} 5 \mathrm{MHz}-\mathrm{BW} 79.7 \mathrm{~dB}-\mathrm{SNDR}$ noiseshaping SAR ADC with dynamic-amplifier-based FIR-IIR filter," IEEE International Solid-State Circuits Conference (ISSCC) Dig. Tech. Papers (2017) 466.

[18] S.-L. Li, et al.: "A 13-ENOB second-order noise-shaping SAR ADC realizing optimized NTF zeros using the error-feedback structure," IEEE J. Solid-State Circuits 53 (2018) 3484 (DOI: 10.1109/ JSSC.2018.2871081).

[19] A. Matsuzawa, et al.: "SAR $+\Delta \Sigma$ ADCs with open-loop integrator using dynamic amplifier," IEICE Electron. Express 15 (2018) 20182002 (DOI: 10.1587/elex.15.20182002).

[20] Z.-J. Chen, et al.: "A 2nd order fully-passive noise-shaping SAR ADC with embedded passive gain," IEEE Asian Solid-State Circuits Conference (A-SSCC) (2016) 309.

[21] W.-J. Guo, et al:: “A 13b-ENOB 173dB-FoM 2nd-order NS SAR ADC with passive integrators," IEEE Symp. VLSI Circuits (2017) C236.

[22] H.-Y. Zhuang, et al.: "A second-order noise-shaping SAR ADC with passive integrator and tri-level voting," IEEE J. Solid-State Circuits 54 (2019) 1636 (DOI: 10.1109/JSSC.2019.2900150).

[23] J.-X. Liu, et al.: "A 40kHz-BW 90dB-SNDR noise-shaping SAR with $4 \times$ passive gain and 2 nd-order mismatch error shaping," IEEE International Solid-State Circuits Conference (ISSCC) Dig. Tech. Papers (2020) (DOI: 10.1109/ISSCC19947.2020.9063159).

[24] Y. Zhu, et al.: "A 10-bit 100-MS/s reference-free SAR ADC in $90 \mathrm{~nm}$ CMOS," IEEE J. Solid-State Circuits 45 (2010) 1111 (DOI: 10.1109/ JSSC. 2010.2048498).

[25] M. Asgari, et al.: "Body effect compensation of analog switches using variable voltage function," IEICE Electron. Express 8 (2011) 189 (DOI: 10.1587/elex.8.189).

[26] H.-M. Chen, et al.: "A high-performance bootstrap switch for low voltage switched-capacitor circuits," IEEE International Symposium on Radio-Frequency Integration Technology (2014).

[27] S. Babayan-Mashhadi, et al.: "Analysis and design of a low-voltage low-power double-tail comparator," IEEE Trans. Very Large Scale Integr. (VLSI) Syst. 22 (2014) 343 (DOI: 10.1109/TVLSI.2013. 2241799).
[28] Z.-J. Chen, et al.: "A 9.35-ENOB, 14.8 fJ/conv.-step fully-passive noise-shaping SAR ADC," IEICE Trans. Electron. E99-C (2016) 963 (DOI: 10.1587/transele.E99.C.963).

[29] W. Guo, et al.: "A 12b-ENOB 61 $\mu \mathrm{W}$ noise-shaping SAR ADC with a passive integrator," IEEE European Solid-state Circuits Conference (2016) 405 (DOI: 10.1109/ESSCIRC.2016.7598327).

[30] Y. Song, et al.: "Passive noise shaping in SAR ADC with improved efficiency," IEEE Trans. Very Large Scale Integr. (VLSI) Syst. 26 (2018) 416 (DOI: 10.1109/TVLSI.2017.2764742). 